\title{
Violencia y convivencia escolar en la región de puno
}

\author{
Yuselino Maquera Maquera ${ }^{1}$ \\ ymmaquera@unap.edu.pe \\ https://orcid.org/0000-0002-7476-9205 \\ Universidad Nacional del Altiplano \\ Puno - Perú \\ Fanny Apaza Gomez ${ }^{2}$ \\ fapazag@epg.unap.edu.pe \\ https://orcid.org/0000-0001-7540-6652 \\ Universidad Nacional del Altiplano \\ Puno - Perú
}

\section{RESUMEN}

La investigación trata sobre la relación de violencia y convivencia escolar en estudiantes de educación secundaria en colegios JEC Jornada Escolar Completa Región de Puno. El objetivo general es.- Determinar la relación entre violencia escolar y convivencia escolar, el estudio es de carácter cuantitativo de tipo básica, el diseño es no experimental de corte transversal, como muestra poblacional es de 120 estudiantes, la técnica utilizada la encuesta y el instrumento es de tipo dicotómico, así mismo el procesamiento estadístico es no paramétrica con el estadígrafo coeficiente de correlación de Rho Spearman. Como conclusión la Violencia escolar se relaciona significativamente en la convivencia escolar, según los resultados obtenidos de la prueba Rho de Spearman = - ,736 dan cuenta de la existencia de una relación negativa media considerable entre las variables de violencia y convivencia escolar; este grado de correlación indica que la relación entre las variables es inversa $\mathrm{p}=0.000$ muestra que $\mathrm{p}$ es menor a 0.05 lo cual permite señalar que la relación es significativa, por lo tanto, se rechaza la hipótesis nula y se acepta la hipótesis alterna.

Palabras Clave: convivencia escolar; violencia escolar; violencia entre pares; violencia institucional

\footnotetext{
1 Doctor, Docente Ordinario en la Universidad Nacional del Altiplano Institución, correo electrónico ymmaquera@unap.edu.pe

${ }^{2}$ Licenciada en Trabajo Social, Maestrante en Trabajo Social con Mención en Promoción de la Familia e Inclusión Social - Universidad Nacional del Altiplano Institución, correo electrónico fapazag@epg.unap.edu.pe
} 


\title{
Violence and school coexistence in the puno region
}

\begin{abstract}
The research deals with the relationship between school violence and school coexistence in students of JEC Full School Day Puno region. Province of San Román District of San Miguel. The general objective is.- To determine the relationship between school violence and school coexistence, the study is of a basic quantitative nature, the design is nonexperimental, cross sectional, as a population sample is 120 students, the technique used in the survey and the The instrument is dichotomous, likewise the statistical processing that was used was non-parametric with the Rho Spearman correlation coefficient statistician. In conclusion, school violence is significantly related to school coexistence, according to the results obtained from the Spearman Rho test =-, 736 show the existence of a considerable negative average relationship between the variables of violence and school coexistence. This degree of correlation indicates that the relationship between the variables is inverse, $p=0.000$ shows that $p$ is less than 0.05 , which allows us to indicate that the relationship is significant, therefore, the null hypothesis is rejected and the alternative hypothesis is accepted.
\end{abstract}

Keywords: school life; school violence; peer violence; institutional violence.

Artículo recibido: 30 noviembre. 2021 Aceptado para publicación: 29 diciembre 2021 Correspondencia: ymmaquera@unap.edu.pe Conflictos de Interés: Ninguna que declarar 


\section{INTRODUCCIÓN}

Es preciso enfatizar, en la mayoría de las instituciones educativas, a nivel mundial se ha depuesto la violencia escolar. Tomando en cuenta a Ospina (2017) la democracia permite que las relaciones de la comunidad educativa se ven en una situación de conflicto, bajo una estructura social desde lo administrativo hasta los estudiantes. De acuerdo con el Fondo de las Naciones Unidas para la Infancia UNICEF (2017) afirma que, a nivel mundial cerca de 130 millones de estudiantes entre las edades de 13 y 15 años, experimentan casos de acoso escolar. La Organización de las Naciones Unidas para la Educación, la ciencia y la cultura UNESCO (2013) indica que dos de cada diez estudiantes en el mundo sufren de acoso. La Organización Mundial de la Salud OMS (2009) manifiesta: "La violencia escolar se presenta de manera grupal o en forma individual. Las edades en la cual se produce de 11 a 17 años, lo cual es considerado como la población en riesgo"(p. 12). Sobre esta postura, la violencia se presenta según la intención del agresor, las causas se presentan según la relación entre la víctima y el agresor, haciendo que esta problemática sea mucho más compleja de lo que se pensaba.

En Perú, al igual a otros Países de la Región, el colegio es el lugar donde los estudiantes pasan una gran parte del día. Las instituciones educativas deben ser espacios acogedores, que propicien la cohesión entre los estudiantes y con una grata convivencia. Según el Ministerio de Educación MINEDU (2010) realza que entre los años 2007 y 2010, mencionó que el 54\% de estudiantes a nivel nacional, manifestó haber sufrido diversas agresiones, el 91\% indicaron haber sufrido por apodos, el 36.5\% sufrieron como código de silencio en clase, el $64 \%$ fueron testigos de estas agresiones, pero optaron por no defender a los agredidos. Estas cifras llevan a reflexionar sobre la función que cumplimos como trabajador social, maestros, como padres o simplemente como personas, que forman parte de esta sociedad. El diario Correo refiere que, entre los años 2013 al 2020, mostraron más de 1300 casos de violencia en el portal, resaltando el $87 \%$ de los casos se presentaron en instituciones educativas públicas, mientras que el 13\% fueron privadas, esto significa que, Lima Metropolitana encabeza la lista de denuncias en su página web, con casi 4 mil casos registrados sobre violencia escolar, en regiones como: Piura se detectaron 828 casos; Junín 618 casos y en Áncash 473 casos, siendo éstos lugares con mayor índice con respecto a esta problemática. (Mendoza, 2019, p. 4) 
Así mismo, al momento de elaborar el PEI (Proyecto Educativo Institucional) de la Institución Educativa Horacio Zevallos Gámez, correspondiente a los años 2018 - 2020, se detectó a través de un FODA (Fortalezas, Oportunidades, Debilidades y Amenazas), el incremento significativo de violencia escolar en las aulas. Este resultado no va en la sintonía que busca el MINEDU (2010) ya que dentro de los compromisos de gestión que propone se encuentra el quinto compromiso que señala la gestión de la convivencia escolar en la Instituciones Educativas y que ha servido como base técnica para esta investigación.

El creciente número de casos de violencia llegó a 2,685 para el año 2018, respecto al año 2020 existen 194, casos reportados en el portal (SíseVe, 2019). Al tratarse de un fenómeno tan amplio, es necesario poder abordarlo en profundidad, existe violencia en el contexto escolar, cuando existe trasgresión brutal del orden escolar, reglas y costumbres en la sociedad. La perspectiva teórica de la investigación, gira en torno a la violencia escolar y convivencia escolar.

El tema de violencia escolar, abordado por (Calle, 2018) es un fenómeno que se presenta en las instituciones educativas y despierta el interés de la comunidad institucional, en tanto, (Ospina, 2017) remarca las formas de violencia que se construye en el ámbito educativo y que afecta el desarrollo autónomo de los sujetos; (Neut, 2017) propone la acción y reacciones en comportamientos individuales; (N. Álvarez, 2015) concibe contextos sociales las configuraciones culturales e importancia les otorgan a los vínculos escolares; (Brandoni, 2017) enfatiza que los vínculos de los miembros de la comunidad educativa son el producto de mecanismos institucionales y pueden acentuar situaciones de violencia social; (UNICEF, 2011) en la comunidad escolar se presentan diferentes tipos de violencia, como la violencia perpetrada por profesores u otros trabajadores de la escuela, puede revestir la forma de castigos corporales, tales como palizas y golpes con vara; por ultimo (Madrigal, 2015) refiere como toda acción, situación o relación que suceda dentro de los límites físicos del establecimiento o en el marco de una relación social, que atenta contra la integridad física, moral, psicológica o social de algún miembro de la comunidad escolar, contra las reglas y normas de la escuela, y cuyo impacto cause dolor o lesiones físicas o psíquicas a las personas que actúan en el ámbito escolar, dañar los objetos que se encuentran en dicho ámbito, afectando la buena convivencia en los centros escolares. 
El siguiente tema es convivencia escolar, el Ministerio de Educación del Perú MINEDU (2018) remarca que es el conjunto de relaciones personales que configuran en la vida escolar; (Muñoz, 2017) la institución educativa es la encargada de promover una convivencia armónica entre los estudiantes, abriendo espacios para aprender el respeto, la tolerancia, la solidaridad, y la inclusión se encarga de generar una convivencia adecuada; (Ortega, 2015) enfatiza el conjunto de interrelaciones entre estudiantes, se llevan a través de normas convencionales en la vida escolar, de respeto mutuo, solidaridad, tolerancia y desarrolla la autoestima,; (Otero, 2016) La importancia de las normas, que sin ellas no es posible el entendimiento entre los miembros de la comunidad escolar, ni se puede llevar a cabo ningún proyecto educativo; (Brandoni, 2017) son constructivos que permiten el crecimiento de los actores sociales, que afecta a las teorías pedagógicas en relación de la convivencia y el aprendizaje, dentro de la organización educativa.

El propósito de la investigación al análisis de la relación que hay entre violencia escolar y convivencia escolar, se pretende indagar la relación sobre las problemáticas ocasionadas por la violencia escolar y los tipos que se ven reflejados en el desarrollo cognitivo que presentan los estudiantes de primero a quinto grado de secundaria de la Institución Educativa Horacio Zevallos.

\section{La presente investigación tuvo como objetivo}

- Determinar la relación entre la violencia escolar y convivencia escolar, como hipótesis se plantea, existe relación significante entre la violencia escolar y convivencia escolar.

\section{ESTRATEGIAS METODOLÓGICAS}

La investigación se desarrolló en la Región de Puno, desde el enfoque cuantitativo. Según (Hernández, 2014) "la investigación cuantitativa usa recolección de datos para probar hipótesis, con base en la medición numérica y el modelo estadístico dicotómico de correlación de factores con la prueba Rho de Spearman. A fin de establecer patrones de comportamientos y probar teorías educativas referentes a convivencia escolar...” (p. 4). El diseño de investigación es no experimental, de corte transversal. Las investigaciones no experimentales "son aquellas que se realizan sin manipular deliberativamente las variables, es decir, se trata de estudios en los que no hacemos variar en forma anexionada las variables, por el contrario se trata de observar fenómenos tal como se dan en su 
contexto natural para analizarlos" (Hernández, 2014, p. 152). La investigación corresponde al tipo básica, este tipo de investigación "están dirigidas a establecer o a refutar teorías y proposiciones fundamentales de una rama de la ciencia" (Rodríguez \& Pérez, 2017). El nivel de la presente investigación corresponde al descriptivo correlacional. Según Hernández (2014), "los estudios correlaciónales responden a preguntas de investigación cuya finalidad es conocer la relación o grado de asociación que existe entre dos o más variables en un contexto particular" (p. 120). El tipo de análisis es RHO Spearman, Los criterios de selección de la población de estudio está conformada por 278 estudiantes entre varones y mujeres, de quinto año de secundaria de las Instituciones JEC en la Región de Puno, han sido registrados en el cuaderno de incidencias en el área de ATI (Atención de Tutoría Integral). Para (Maquera \& Apaza, 2021) ha puesto en alerta al Ministerio de Educación y en esta coyuntura se crea las instituciones educativas con Jornada Escolar Completa JEC, con la intervención de los profesionales de Trabajo Social y Psicología desde el 2015. Para la selección de la muestra de estudio se utilizó el muestreo probabilístico y aleatorio simple, el mismo que consistió en elegir estudiantes reportados en la plataforma (SíseVe, 2019) tomando los siguientes criterios: Beneficiario del ATI, estudiante registrado en libro de incidencias, estudiantes atendido por el trabajador social según caso derivado por el docente tutor, estudiante reportado por el auxiliar, que hacen un total de 102 estudiantes de quinto año registrados en la plataforma SISEVE.

\section{RESULTADOS Y DISCUSIÓN}

\section{Tabla 1}

Relación de la violencia entre pares según la Convivencia Escolar de estudiantes de colegios JEC en la Región de Puno, 2020.

\begin{tabular}{|c|c|c|c|c|c|}
\hline & & & \multicolumn{2}{|c|}{ Convivencia Escolar } & \multirow{2}{*}{ TOTAL } \\
\hline & & & NO & SI & \\
\hline \multirow{4}{*}{$\begin{array}{c}\text { Violencia } \\
\text { Entre Pares }\end{array}$} & \multirow{2}{*}{ SI } & $\mathrm{n}^{\circ}$ & 4 & 10 & 14 \\
\hline & & $\%$ & $3,9 \%$ & $9,8 \%$ & $13,7 \%$ \\
\hline & \multirow{2}{*}{$\mathrm{NO}$} & $\mathrm{n}^{\circ}$ & 9 & 79 & 88 \\
\hline & & $\%$ & $8,8 \%$ & $77,5 \%$ & $86,3 \%$ \\
\hline \multirow{2}{*}{ TOTAL } & & $\mathrm{N}$ & 13 & 89 & 102 \\
\hline & & $\%$ & $12,7 \%$ & $87,3 \%$ & $100,0 \%$ \\
\hline
\end{tabular}

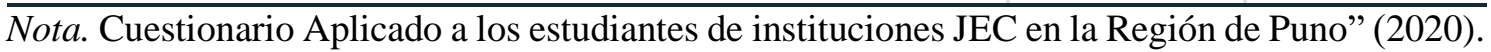


El 77,5 \% de los estudiantes manifiestan que "si" mantienen una adecuada convivencia escolar y "no" sufren de violencia entre pares. Por lo tanto, se ha considerado los indicadores como interacción con los demás, comunicación y correspondencia, el respeto, comportamiento y puntualidad con buenas relaciones interpersonales dándose una comunicación adecuada entre compañeros. Así mismo, señalar que el 3,9\% de estudiantes encuestados, manifiestan que "no" mantienen la convivencia escolar adecuada, pero "si" son sujetos de violencia entre pares. En esta dimensión se ha considerado los indicadores como intimidación y/o hostigamiento, inseguridad y la exclusión social, entre estos tres indicadores los estudiantes, señalaron que la violencia entre pares se presenta más por la inseguridad. Según Madrigal (2015) refiere que en las instituciones existen otros estudiantes que se sienten inseguros de lo que hacen y también sienten que son discriminados, así mismo (Bermúdez et al., 2021) refieren que los estudiantes presentan niveles de depresesión y ansiedad que son obtenidos por factores psicosociales.

La Teoría instintiva de la violencia escolar es una tendencia innata como menciona Freud (2003), sostiene que es el instinto de muerte o thanatos el que estaría a la base de esta conducta. Dicho instinto sería inicialmente de autodestrucción y posteriormente se volcaría hacia el entorno de los individuos. Por otra parte, consideró que la agresividad surge, principalmente, del "instinto de lucha", que se habría desarrollado en la especie para dispersar a la población en grandes áreas, a la vez que para asegurar la reproducción de los más “fuertes”, quienes pasarían su carga genética a las siguientes generaciones.

\section{Tabla 2}

Relación de la violencia Institucional según la Convivencia Escolar de estudiantes de colegios JEC en la Región de Puno, 2020

\begin{tabular}{|c|c|c|c|c|c|}
\hline & & & \multicolumn{2}{|c|}{ Convivencia Escolar } & \multirow{2}{*}{ TOTAL } \\
\hline & & & NO & SI & \\
\hline \multirow{4}{*}{$\begin{array}{c}\text { Violencia } \\
\text { Institucional }\end{array}$} & \multirow{2}{*}{ SI } & $\mathrm{n}^{\circ}$ & 3 & 11 & 14 \\
\hline & & $\%$ & $2,9 \%$ & $10,8 \%$ & $13,7 \%$ \\
\hline & \multirow{2}{*}{ NO } & $\mathrm{n}^{\circ}$ & 10 & 78 & 88 \\
\hline & & $\%$ & $9,8 \%$ & $76,5 \%$ & $86,3 \%$ \\
\hline \multirow{2}{*}{\multicolumn{2}{|c|}{ TOTAL }} & $\mathrm{N}$ & 13 & 89 & 102 \\
\hline & & $\%$ & $12,7 \%$ & $87,3 \%$ & $100,0 \%$ \\
\hline
\end{tabular}

Nota. Cuestionario Aplicado de instituciones JEC en la Región de Puno. (2020). 
El 76,5 \% de estudiantes manifiestan tener una convivencia escolar adecuada, representado por "si" los estudiantes poseen buenas relaciones interpersonales, manejan las normas de convivencia que están estructuradas a través de un orden escolar. Por su parte, los estudiantes indican que sufren violencia institucional representada por el "no". Así mismo el 2,9\% no tienen una convivencia adecuada, por tanto que "si" sufren de violencia institucional. En esta dimensión se ha considerado los indicadores como abuso de poder, calidad de proceso de enseñanza, actitudes de decidía de directivos y el castigo físico, entre estos cuatro indicadores, los estudiantes han señalado, que son sujetos a la violencia institucional porqué tuvieron problemas con su docente, fue sujeto de insulto, amenaza por parte de los docentes. Por lo tanto (Maquera et al., 2020) refieren que transformación de las instituciones, propician la generación de nuevos modelos educativos para atender al perfil de las generaciones que ingresan, con el objetivo de desarrollar las competencias que les servirán de herramienta para hacer frente a los retos que se les presentan.

\section{Tabla 3}

Relación de violencia en torno hacia la escuela según la Convivencia Escolar de estudiantes de colegios JEC en la Región de Puno, 2020.

\begin{tabular}{|c|c|c|c|c|c|}
\hline & & & \multicolumn{2}{|c|}{ Convivencia Escolar } & \multirow{2}{*}{ TOTAL } \\
\hline & & & NO & SI & \\
\hline \multirow{4}{*}{$\begin{array}{c}\text { Violencia en torno hacia } \\
\text { la Escuela }\end{array}$} & \multirow{2}{*}{$\mathrm{SI}$} & $\mathrm{n}^{\circ}$ & 1 & 9 & 10 \\
\hline & & $\%$ & $1,0 \%$ & $8,8 \%$ & $9,8 \%$ \\
\hline & \multirow{2}{*}{ NO } & $\mathrm{n}^{\circ}$ & 12 & 80 & 92 \\
\hline & & $\%$ & $11,8 \%$ & $78,4 \%$ & $90,2 \%$ \\
\hline \multirow{2}{*}{\multicolumn{2}{|c|}{ TOTAL }} & $\mathrm{N}$ & 13 & 89 & 102 \\
\hline & & $\%$ & $12,7 \%$ & $87,3 \%$ & $100,0 \%$ \\
\hline
\end{tabular}

Nota. Cuestionario Aplicado de instituciones JEC en la Región de Puno” (2020).

El 78,4 \% de estudiantes manifiestan que "si" mantiene una convivencia adecuada y "no" sufren de violencia en torno hacia la escuela, los estudiantes poseen buenas relaciones interpersonales, práctica de valores y normas de convivencia.

Así mismo el 1.0\% refiere que "si" es sujeto de violencia en torno hacia la escuela y "no" tiene una convivencia. En esta dimensión se ha considerado los indicadores como ciberbulling, grooming, sexting y sextorsion, entre estos cuatro indicadores los 
estudiantes señalan que existen agresores que envían mensajes vía mecanismos electrónicos como Facebook, Instagram, WhatsApp, tiktok, Youtube, y otras redes sociales donde se practica la discriminación, con contenidos pornográficos, bullying y ciberbullying.

La Teoría del impulso, desarrollada por Dollard \& Miller (1950), plantea que la agresividad nace de un impulso desde el exterior y que lleva a dañar a los demás, fundamentalmente en la autoestima, en el honor, la reputación y el vejamen, que a fin de cuentas viene a dañar la integridad moral y psicológico, víctima del acoso escolar para (Vásquez, 2018) la nueva dinámica se presenta con la vertiginosa presencia de información en todos los dominios de la vida y quehacer social, la sofisticación tecnológica que permite encontrarlos, reproducirlos y tener acceso a ellos, y la necesidad de mayor especialización de quienes trabajan con conocimientos, llegando hasta los niveles de depresión y frustración en estudiantes adolescentes en proceso de formación escolar. Por estas consideraciones el Ministerio de Educación creó el plan de convivencia escolar, (Paredes \& Vásquez, 2018) la situación en las que las TICs permitieron al Trabajador Social realizar una intervención ágil y de calidad, generando un impacto positivo en la sociedad, contribuyendo al bienestar social y a posicionarnos como verdaderos agentes de cambio, cuya apertura e intervención está a la vanguardia y a la altura de las circunstancias.

\section{CONSIDERACIONES FINALES}

De acuerdo a los resultados del análisis estadístico dan cuenta de la existencia de una relación negativa débil considerable según Rho de Spearman = -,225* entre la dimensión de violencia entre pares y convivencia escolar. Este grado de correlación indica que la relación entre las variables es inversa y tienen un nivel de correlación negativa débil. En efecto coincidimos con las investigaciones de (Madrigal, 2015; Neut, 2017; Rodríguez, 2018) se muestra que la violencia es alta la disrupción en el aula y exclusión social, se puede notar que los estudiantes señalaron haber sido discriminados, por su condición social de pobreza y cultura .También se encontró que estos resultados coinciden con la teoría de la interacción de Ramos, quien manifestó que , el poder económico de algunos y la de pobreza de otros, tienden a dañar valores de los individuos.

De acuerdo a los resultados del análisis estadístico dan cuenta de la existencia de una relación negativa media considerable según Rho de Spearman =-,563**entre la 
dimensión y la variable de violencia institucional. Este grado de correlación indica que la relación entre las variables es inversa y tienen un nivel de correlación negativa media. De acuerdo con la investigaciones de (Chirinos, 2019; Flores, 2019; Mercedes, 2015) refieren que los tipos de violencia escolar ocurren con mayor frecuencia son como la disrupción en el aula, violencia verbal de alumno- alumno, violencia física directa y amenaza entre estudiantes, lo de menor frecuencia del profesorado hacia el alumno. Así mismo coincidimos con en su investigación menciona que la dimensión de violencia institucional es un incidente que provoca la debilidad en convivencia escolar.

De acuerdo a los resultados del análisis estadístico dan cuenta de la existencia de una relación negativa media considerable según Rho de Spearman $=-, 603 * *$ entre la dimensión de violencia en torno hacia la escuela y la variable de convivencia. Este grado de correlación indica que la relación entre las variables es inversa y tienen un nivel de correlación negativa considerable. En efecto coincidimos con (Álvarez, 2015; Hidalgo \& Ramirez, 2013; P. Rodríguez, 2018) en sus investigaciones según el tipo de violencia escolar indica notoriamente que la diferencia de género, determina que tan violento puede ser el ser humano, pero al momento de especificar según el tipo de violencia en torno hacia la escuela significa que afuera de cada institución muchos adolescentes sufren el cyberbulling, como la tecnología ha provocado en los adolescentes, en sus investigaciones las dimensiones que mencionan acerca de las tecnologías de la información y de la comunicación indican que el $45 \%$ la mayoría de estudiantes sufren este tipo de violencia en torno hacia la escuela, estos datos concuerda con los testimonios $\mathrm{y}$ vivencias de los estudiantes que señalan $28 \%$ sufren la violencia en torno hacia la escuela, entendida también como violencia fuera del espacio escolar.

Frente a esta problemática de violencia escolar en sus diversas manifestaciones: La violencia entre pares; violencia institucional; violencia en torno hacia la escuela, existen 102 casos que testifican que han sido víctimas de estos tipos de violencia registrados en la plataforma del SISEVE de cada institución. El trabajador social diseña un conjunto de proyectos y programas destinados a generar una convivencia pacífica dentro y fuera de la institución, prover una cultura de paz, con un conjunto de estrategias que pasa por procesos de capacitación, asistencia social, promoción social y estudios de casos, que contribuyen a mediar conflictos al interior de las instituciones educativas en la Región de Puno. 
Violencia y convivencia escolar...

\section{LISTA DE REFERENCIAS}

Álvarez, E. (2015). Violencia Escolar : Variables Predictivas en Adolescentes Gallegos. Universidad de Vigo. [Tesis para optar el grado de Doctor en Ciencias de la Educación, Univesidad de Vigo]. https://digibuo.uniovi.es/dspace/bitstream/handle/10651/39317/TD_JosefinaMar garitaMercedes.pdf;jsessionid=1D5FFA942A973BB98037CFDC4F9C71CC?se quence.

Álvarez, N. (2015). La violencia escolar en perspectiva histórica. Universidad de Buenos Aires Facultad de Filosofía y Letras.

Bermúdez, C. A., García, M. S., \& Vasquez, M. J. (2021). Psychosocial factors predicting resilience in family caregivers of children with cancer: A cross-sectional study. International Journal of Environmental Research and Public Health, 18(2), 1-13. http://doi.org/10.3390/ ijerph18020748

Brandoni, F. (2017). Conflictos en la escuela (Eduntref). http://eduntref.com.ar/magento/pdf/conflictos-en-la-escuela-digital.pdf

Calle, G. (2018). Estrategia formativa para mitigar la violencia escolar en perspectiva de derechos humanos. Educación y Humanismo, 20(34), 79-95. http://dx.doi.org/10.17081/eduhum.20.34.2859

Chirinos, D. (2019). «Percepción de la violencia escolar en adolescentes de una institución educativa estatal en Santa Anita. Lima-Perú2019». Universidad Nacional Mayor de San Marcos.

Dollard, J., \& Miller, N. (1950). El analisis experimental de la conducta. En Teoria psicoanalitica del aprendizaje 201227).https://www.revistahistoriapsicologia.es/

Flores, F. (2019). Violencia escolar y convivencia social en niñas de 10 a 12 años del colegio José María Arguedas, Lima 2019 [Tesis para el titulo Profesional de licenciada en Enfermería,Universidad César Vallejo]. https://repositorio.ucv.edu.pe/handle/20.500.12692/38299

Freud, S. (2003). Psicoanálisis. https://doi.org/10.4324/9780203361825

Hernández, R. (2014). Metodología de la Investigación (Sexta).

Hidalgo, D., \& Ramirez, K. (2013). Tipos De Violencia Escolar En Alumnas Y Alumnos De Séptimo Años Básico Según Género [Tesis para optar la segunda titulación en 
Educación, Universidad Academia de Humanismo Cristiano de Santiago]. http://bibliotecadigital.academia.cl/bitstream/handle/123456789/1097/tpedif 14.pdf? sequence $=3 \&$ is Allowed $=y$

Madrigal, A. (2015). Violencia escolar en México : una exploración de sus dimensiones y consecuencias. Trabajo social, 23-42. http://www.revistas.unam.mx/index.php/ents/article/view/54048

Maquera, Y., \& Apaza, F. (2021). Trabajo social, violencia y promoción de la convivencia escolar en la Región de Puno. En Cultura y pedagogía de la paz, estudios realizados en México y Latinoamérica (p. 369). https://uhjs.edu.mx/index.php/editoral/3-educacion/3-cultura-y-pedagogia-de-lapaz-estudios-realizados-en-mexico-y-latinoamerica

Maquera, Y., Calderon, A., \& Alanoca, C. (2020). Educación universitaria y el proceso de homogenización en el Surandino (Peruano). En Los paradigmas actuales educación, empresa y sociedad (p. 671). https://editorialeidec.com/producto/losparadigmas-actuales-educacion-empresa-y-sociedad/

Mendoza, R. (2019). Advierten que el 38\% de colegios no tiene protocolos para tratar violencia. correo. https://diariocorreo.pe/peru/advierten-que-el-38-de-colegiosno-tiene-protocolos-para-tratar-violencia-926334/

Mercedes, J. (2015). Evaluación de la Convivencia Escolar en los Centros Educativos de Santo Domingo [Tesis doctoral, Universidad de Oviedo de Republica Dominicana].

https://digibuo.uniovi.es/dspace/bitstream/handle/10651/39317/TD_JosefinaMar garitaMercedes.pdf;jsessionid=1D5FFA942A973BB98037CFDC4F9C71CC?se quence $=1$

MINEDU. (2010). Educación Básica Regular. MInisterio de Educación, 1-478. https://doi.org/10.1257/app.20130267

MINEDU, (Ministerio de educación). (2018). Lineamientos para la gestión de la convivencia escolar, la prevención y la atención de la violencia contra niñas, niños y adolescentes.

Muñoz, F. (2017). Elaboración y validación psicométrica del cuestionario de convivencia escolar para la no violencia (CENVI). Estudios Pedagógicos, XLIII, $N^{\circ}(3), 205-$ 223. https://doi.org/10.4067/S0718-07052017000300012 
Neut, P. (2017). Las violencias escolares en el escenario educativo chileno. Análisis crítico del estado del arte. Calidad en la Educación, 46, 222. https://doi.org/10.31619/caledu.n46.8

OMS. (2009). Informe mundial sobre la violencia y la salud. Revista do Instituto de Medicina Tropical de São Paulo, 45(3), 130-130. https://doi.org/10.1590/s0036$\underline{46652003000300014}$

Ortega, R. (2015). Convivencia escolar : fortaleza de la comunidad educativa y protección ante la conflictividad escolar. Interuniversitaria de formación del profesorado, 66(23), 159-180.__ https://doi.org/file:///C:/Users/intel/Downloads/DialnetConvivenciaEscolar-3098226.pdf

Ospina, Y. (2017). Nuevas miradas de la violencia escolar retos y desafíos para los docentes. Educación $\quad \& \quad$ Pensamiento, https:file:///C:/Users/intel/Downloads/Dialnet-

NuevasMiradasDeViolenciaEscolar-6178580.pdf

Otero, V. (2016). Convivencia escolar problemas y soluciones. Complutense de Educación, $12(1)$, 295-318. https://revistas.ucm.es/index.php/RCED/article/view/17762

Paredes, A. M., \& Vásquez, M. J. (2018). Tecnologias de la Informacion y Comunicación un Trabajo de Acercamiento Desde Las Ciencias Sociales, Una Experiencia Desde El Trabajo Social. Big Bang Faustiniano, 7(4), 1-4. https:file:///C:/Users/intel/Downloads/500-Texto\%20del\%20art\%C3\%ADculo1376-1-10-20191108.pdf

Rodríguez, A., \& Pérez, A. (2017). Métodos científicos de indagación y de construcción del conocimiento. Revista EAN, 82, 179-200. https://doi.org/10.21158/01208160.n82.2017.1647

Rodríguez, P. (2018). Violencia escolar en estudiantes de tercero, cuarto y quinto grado de secundaria de la Institución Educativa Parroquial "San Martín de Porres"Acarí [Tesis para optar el Titulo Profesional de Licenciado en PsicologiA, Universidad Inca Gracilaso de la Vega en Lima]. http://repositorio.uigv.edu.pe/bitstream/handle/20.500.11818/2372/TRAB.SUF.P ROF.Paulino\%20Rodr\%c3\%adguez\%20V\%c3\%a1squez.pdf?sequence=2\&isAll owed $=\mathrm{y}$ 
SíseVe, (Sistema Especializado en reporte de casos sobre violencia Escolar). (2019). Total de casos Reportados.http://www.siseve.pe/Web/file/materiales/InformeMemorias-S\%C3\%ADseve.pdf

UNESCO, (Organización de las Naciones Unidas para la Educación, la C. y la C. (2013). Respuestas del Sector de la Educación al Bullying Homofóbico. En Oficina Regional de Educación para América Latina y el Caribe (pp. 1-70). http://www.unesco.org/new/fileadmin/MULTIMEDIA/FIELD/Havana/pdf/Educ acion_bullying.pdf

UNICEF, (Fondo de las Naciones Unidas). (2011). Violencia escolar en América Latina y el Caribe (Clayton). https://repositorio.cepal.org/bitstream/handle/11362/41068/4/S1700122_es.pdf

UNICEF, (Fondo de las Naciones Unidas). (2017). Una situación habitual. Violencia en las vidas de los niños y los adolescentes. American Journal of Criminal Justice, Vol 40, $n^{\circ}, 1-22$.

Vásquez, M. J. (2018). Aspectos socio jurídicos de gestión victimológica. Estudio de una unidad de asistencia a victimas en el Distrito Fiscal de Arequipa-2018 [Tesis para optar el Grado Académico de Maestro. Universidad Nacional de San Agustin Arequipa].

http://eprints.rclis.org/41892/1/Aspectos\%20Socio\%20juridicos\%20gestion\%20 victimologica\%20gerencia\%20victima\%20trabajo\%20social\%20intervencion\%

20social\%20comunitaria.pdf 